\title{
Associação de laser de baixa potência e ácido hialurônico no tratamento de dermatite de contato alérgica: um relato de caso
}

\author{
Asociación de láser de baja potencia y ácido hialurónico en el tratamiento de la dermatitis \\ alérgica de contacto: reporte de un caso \\ Association of low-power laser and hyaluronic acid in the treatment of allergic contact dermati- \\ tis: a case report
}

\section{Resumo}

Objetivos: Este estudo tem por objetivo relatar a efetividade do laser de baixa potência (LBP) associado à terapia tópica com Ácido Hialurônico (AH) 0,2\% em dermatite de contato alérgica. Metodologia: Trata-se de estudo descritivo exploratório de relato de experiência com paciente assistida em domicílio por uma enfermeira especialista em feridas em uma cidade do interior paulista. Preconizou-se protocolo com aplicação a cada 48 horas de LBP combinado com administração tópica diária de $\mathrm{AH}$ a 0,2\%. A análise dos efeitos do tratamento foi realizada por meio de registro fotográfico e as imagens foram analisadas com auxilio do software Imagej. Os curativos foram realizados diariamente e, em 17 dias, observou-se a total cicatrização da lesão. Resultados: Ao final de 17 dias a paciente apresentou cicatrização completa. cicatrização completa, e considerando o fototipo cutâneo da paciente, a mesma foi orientada a dar continuidade ao tratamento diário com aplicação tópica de $\mathrm{AH} \mathrm{0,2 \%} \mathrm{por} \mathrm{mais} 15$ dias, visando a melhora da estética do processo cicatricial. Conclusão: Concluiu-se que a terapêutica proposta apresentou boa tolerabilidade e eficácia para este caso em particular.

Descritores: Ácido hialurônico, dermatite de contato alérgica, laser de baixa potência.

\section{Abstract}

Objectives: This study aims to report the effectiveness of low power laser (LPL) associated with topical therapy with $0.2 \%$ Hyaluronic Acid $(\mathrm{HA})$ in allergic contact dermatitis. Methodology: This is an exploratory descriptive study reporting an experience with a patient assisted at home by a wound specialist nurse in a city in the interior of São Paulo. A protocol with application every 48 hours of LPL combined with daily topical administration of $0.2 \%$ HA was recommended. The analysis of

\section{Marilene Neves da Silva}

Doutora em Clínica Médica (Dermatologia Clínica e Molecular) pela Universidade Estadual de Campinas (Unicamp). Mestre em Ciências Biomédicas. Graduada em Enfermagem. Membro da Sociedade Brasileira de Estomaterapia (Sobest). Atualmente desenvolve projeto em parceria com - Departamento de Dermatologia da University of Minnesota (USA). ORCID: 0000-0002-0885-1083

\section{Gislaine Vieira-Damiani}

Possui graduação em Ciências Biológicas Modalidade Médica (Bacharelado e Licenciatura), mestrado (2009) e doutorado (2012) em Fisiopatologia Medica -Universidade Estadual de Campinas (2009). Pós-doutorado em Clinica Médica na Universidade Estadual de Campinas. Estudo da matriz extra celular (colágeno) e Diagnóstico de Bartoneloses humana. Professora do Instituto Federal de São Paulo. ORCID: 0000-000 1-8036-6616

Valéria Aparecida Masson Doutora em enfermagem (2012), mes- 
the effects of the treatment was carried out by means of a photographic record and the images were analyzed using the Imagel software. The dressings were applied daily and, in 17 days, the lesion had completely healed. Results: After 17 days, the patient had complete healing. complete healing, and considering the patient's skin phototype, she was advised to continue the daily treatment with topical application of $0.2 \% \mathrm{HA}$ for another 15 days, aiming at improving the esthetics of the healing process. Conclusion: It was concluded that the proposed therapy presented good tolerability and efficacy for this particular case.

Descriptors: hyaluronic acid, allergic contact dermatitis, low-level laser therapy

\section{Resumen}

Objetivos: Este estudio tiene como objetivo reportar la efectividad del láse de baja potencia (LBP) asociado a la terapia tópica con Ácido Hialuróni co $(\mathrm{AH})$ al $0.2 \%$ en la dermatitis alérgica de contacto. Metodología: S trata de un estudio descriptivo exploratorio que reporta una experiencia con un paciente asistido en su domicilio por una enfermera especialista en heridas en una ciudad del interior de São Paulo. Se recomendó un pro tocolo con aplicación cada 48 horas de dolor lumbar combinado con la administración tópica diaria de HA al 0,2\%. El análisis de los efectos de tratamiento se realizó mediante un registro fotográfico y las imágenes se analizaron mediante el software Imagej. Los apósitos se aplicaron diaria mente y, en 17 días, la lesión había cicatrizado por completo. Resultados: Después de 17 días, el paciente tuvo una curación completa. cicatriza ción completa, y considerando el fototipo de piel de la paciente, se le recomendó continuar el tratamiento diario con aplicación tópica de $\mathrm{HA}$ a 0,2\% durante otros 15 días, con el objetivo de mejorar la estética del pro ceso de cicatrización. Conclusión: Se concluyó que la terapia propuesta presentó buena tolerabilidad y eficacia para este caso en particular.

Descriptores: ácido hialurónico, dermatitis alérgica de contacto, láser de baja potencia

RECEBIDO 16/07/2021 | APROVADO: 17/10/2021

\section{INTRODUÇ̃̃O}

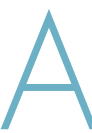

pele é o maior órgão do corpo humano, identificada como órgão periférico do sistema imune por manifestar mecanismos próprios, cujas propriedades de defesa variam de acordo com o local e sua densidade 1. A Pode ser considerada o primeiro agente imunológico identificado no organismo humano, variando de acordo com sua permeabilidade, localização anatômica, espessura, espaço físico onde se encontra e seu potencial de reação imunológica 2.

A dermatite de contato é uma reação inflamatória na pele, decorrente da exposição a um agente capaz de causar irritação ou alergia. Existem dois tipos de dermatite de contato: a irritativa e a alérgica. A irritativa é causada por substâncias ácidas ou alcalinas, como sabonetes, detergentes tre em enfermagem (2009) bacharel e licenciada em enfermagem pela Universidade Estadual de Campinas (2005). Atualmente, participa do Grupo de Estudos e Pesquisas em Saúde e Trabalho da Faculdade de Enfermagem da Universidade Estadual de Campinas. Especialista em saúde do trabalhador (2008). Especialista em Enfermagem em Estomaterapia ORCID: 0000-0002-5076-635X

\section{Pedro Gonçalves de Oliveira}

Graduado em Farmácia e Bioquímica pela Faculdade de Ciências Farmacêuticas Oswaldo Cruz - FCF-FOC (1992), com Doutorado em Fármaco e Medicamentos pela FCF-USP (2002). Possui Especialização em Fitoterapia pelo Instituto Brasileiro de Estudos Homeopáticos - IBEHE/UNAERP (1996), Especialização em Economia e Gestão em Saúde - CPES/UNIFESP (2002), Especialização em Cuidados Paliativos e Terapia da Dor - PUC Minas (2019) e Aperfeiçoamento em Vigilância Sanitária - ENSP/FIOCRUZ (2005) e International Trader - ABRACOMEX (2011).

\section{ORCID: 0000-0001-9853-0031}

\section{João Cezar Castilho}

Graduação em Farmácia Industrial, mestrado em Farmacologia pela Universidade Federal do Paraná e especialização em Farmácia Estética. Atualmente é farmacêutico responsável pela área de produtos para saúde e atua como coordenador de Pesquisa e Desenvolvimento da TRB Pharma. ORCID: 0000-0002-8649-1714

ou solventes, entre outras. Pode aparecer na primeira vez em que entramos em contato com o agente causador o que ocorre com um grande núme- 
ro de pessoas. As lesões geralmente são restritas ao local do contato, já a dermatite alérgica surge após repetidas exposições ao agente agressor e depende de ações do sistema de defesa do organismo. Por esse motivo, pode demorar de meses a anos após o contato inicial para ocorrer 3. Em peles sensíveis, a Dermatite de Contato Alérgica (DCA) pode se manifestar ao primeiro contato a agentes agressores que, ao entrarem em contato com o tecido epitelial, ativam mecanismos imunológicos do indivíduo, que acabam lesionando a pele 4 .

A inflamação da DCA, que também pode ser denominada como Hipersensibilidade de Contato (HSC), se desenvolve a partir da atuação imunológica do indivíduo. Pode ser originada por produtos de natureza orgânica ou inorgânica, retirados de plantas ou de agentes sintéticos 5. Inicialmente, a DCA se caracteriza muito pruriginosa, com edema local, eritema e visível demarcação da dermatite, às vezes dolorosa, podendo apresentar bolhas e até necrose 1.

No mercado, existe uma imensa diversidade de coberturas à disposição para os diferentes estágios de cicatrização. Nessa conjuntura, destacam-se produtos à base de ácido hialurônico $(A H)$, enquanto tecnologia útil para o tratamento de lesões pele 6. $\mathrm{OAH}$ é um biopolímero formado por repetições de um dissacarídeo composto pelo ácido D-glucurônico e a N-acetilglicosamina. Faz parte da constituição do organismo e atua no preenchimento dos espaços intracelulares 7. Apresenta características essenciais a qualquer cobertura de origem biológica, como biocompatibilidade, biodegradabilidade e não indução de imunogenicidade 8 .

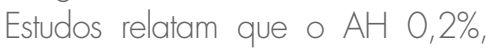
além de acelerar o processo de cica-
A associação de uma cobertura adequada com a fotobiomodulação com laser de baixa potência (LBP), como técnica adjuvante no tratamento de feridas, tem se mostrado eficiente na potencialização do processo de cicatrização. Estudos apontam que a Terapia a Laser de Baixa Potência (TLBP) apresenta efeitos fotoquímicos, fotofísicos e fotobiológicos capazes de alterar

o comportamento celular e favorecer

a reparação tecidual trização, é de fácil manuseio e pode ser aplicado tanto no leito da lesão quanto na área adjacente, o que têm contribuído para a aderência ao tratamento 9,10,1 1 .

A associação de uma cobertura adequada com a fotobiomodulação com laser de baixa potência (LBP) como técnica adjuvante no tratamento de feridas, tem se mostrado eficiente na potencialização do processo de cicatrização. Estudos apontam que a Terapia a Laser de Baixa Potência (TLBP) apresenta efeitos fotoquímicos, fotofísicos e fotobiológicos capazes de alterar o comportamento celular e favorecer a reparação tecidual 12, 13,14.

Este estudo tem por objetivo relatar a efetividade da TLBP associada à terapia tópica com $\mathrm{AH}$ 0,2\% em pacientes com DCA a poliéster avaliada por enfermeira especialista em feridas em domicílio.

\section{METODOLOGIA}

Trata-se de um estudo descritivo exploratório de relato de experiência envolvendo paciente assistida em domicílio no interior paulista.

Paciente do sexo feminino, 45 anos, branca, solteira, sem históricos de alergias ou hipersensibilidade na família. Foi diagnosticada com alergia a poliéster em 03 de março de 2021 em virtude de queixa de prurido intenso e erupção cutânea.

Considerando que a lesão não apresentava nenhum processo infeccioso, iniciou-se o protocolo com a limpeza diária com solução salina, aplicação tópica de creme contendo $\mathrm{AH} \mathrm{O,2 \%} 1$ vez ao dia, e aplicação de Terapia a Laser de Baixa Potência (TLBP) a cada dois dias. $\bigcirc$ protocolo foi mantido por 8 dias.

A coleta de dados foi realizada diariamente entre os dias $\mathrm{O} 3$ e $20 \mathrm{de}$ 


\section{Figura 1: Evolução da lesão da paciente analisada.}

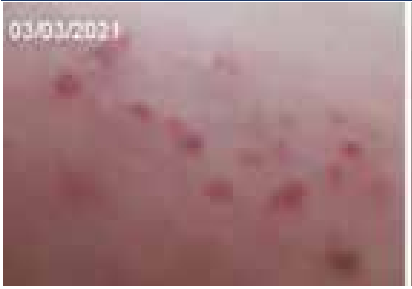

março de 2021, mediante aplicação de um instrumento de avaliação que abrangia as seguintes informações: dados de identificação lidade, sexo, ocupação, diagnóstico); avaliação da lesão (tipo, localização, conteúdo microbiano, exsudato, bordas, pele adjacente, dor, mensuração) e espaço para observações adicionais que se fizessem necessárias. A evolução da lesão foi acompanhada diariamente, avaliada e registrada por uma enfermeira especialista em feridas. Foram realizados registros fotográficos da lesão, empregando-se câmera digital com resolução de 16 megapixels e a uma distância padronizada de 20 centímetros. As imagens foram analisadas com auxilio do software Image 15.

A paciente foi informada a respeito da pesquisa sobre os objetivos e procedimentos envolvidos. $\bigcirc$ termo de autorização de uso de imagem foi assinado pela paciente conforme preconizado pela Resolução 466/2012 do Conselho Nacional de Saúde.

\section{RESULTADOS}

No dia 03 de março de 2021, foi realizada a primeira avaliação da paciente. Nesta ocasião, identificou-se que o ferimento foi decorrente de dermatite de contato por fricção, apresentando prurido intenso na região da mama direita, quadrante inferior direito, o que culminou em DCA. Nessa avaliação, verificou-se presença de lesão com leito eritematoso e lesões mi-
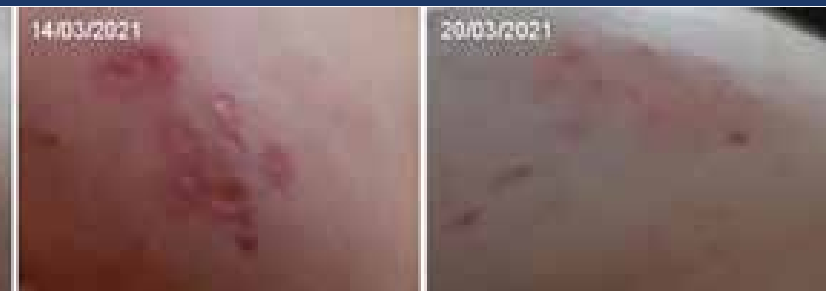

crovesiculares com pequena quantidade de exsudato. As medidas lineares da ferida foram: $5 \mathrm{~cm}$ de base $\times 3 \mathrm{~cm}$ de altura (Figura 1A).

$\bigcirc$ protocolo inicial teve por objetivo reparar o leito da lesão e reduzir a erupção cutânea, fazendo uso de terapia tópica à base de $\mathrm{AH} \mathrm{0,2 \%}$ (Hyaludermin $®$ - TRB Pharma) em fina camada e com trocas diárias. Ao final de sete dias (10/03/2021), houve significativa redução das lesões microvesiculares em base eritematosa com tecido de granulação predominando em grande parte do leito da lesão (Figura 1B). Assim, iniciou-se novo protocolo com TLBP combinado ao $\mathrm{AH}$ $0,2 \%$. Para tanto, empregou-se, laser vermelho AsGaAl (Arseneto Gálio Alumínio) com densidade de energia de 1 j/cm2 no leito da lesão, com tempo calculado de acordo com a fórmula $T=D E \times A / P$, em que $T$ consiste no tempo de aplicação, em segundos; DE significa densidade de energia em joules/cm2 e P corresponde à potência do aparelho em watts.

† tempo de aplicação foi estabelecido em 40 segundos e a forma de aplicação do LBP foi em varredura. Após 96 horas, ao observar a quantidade de tecido de granulação e methora do aspecto cicatricial, optou-se pela manutenção da terapia tópica à base de $A H$ 0,2\% associada a TLBP. (Figura 1C).

No dia 20/03/2021, durante a consulta de enfermagem, constatou-se cicatrização completa, com 100\% de tecido de epitelização (Figura 1D). A paciente evoluiu com medidas lineares satisfatórias, o que resultou na alta do serviço de enfermagem.

Mesmo após cicatrização completa, e considerando o fototipo cutâneo da paciente, a mesma foi orientada a dar continuidade ao tratamento diário com aplicação tópica de $\mathrm{AH}$ 0,2\% por mais 15 dias, visando a melhora da estética do processo cicatricial. Não foram observados desconfortos ou complicações decorrentes do uso do protocolo empregado.

\section{CONCLUSÃO}

Em virtude das DCAs apresentarem mediação do sistema imune, as mesmas devem ter controle baseado no distanciamento do agente irritante. Caso isso não seja possível, deve-se considerar outras medidas terapêuticas. $\bigcirc$ ácido hialurônico (AH) vem alcançando visibilidade como uma nova tecnologia para o tratamento de lesões dérmicas e epidérmicas. A TLBP tem se mostrado uma excelente terapia coadjuvante ao tratamento de lesões de pele, sua ação tem proporcionado a liberação de fatores de crescimento, melhora do fluxo sanguíneo e consequentemente a aceleração do processo inflamatório. Os resultados descritos neste relato de experiência sugerem que a associação desses dois recursos terapêuticos pode apresentar resultados promissores no tratamento de DCAs. 


\section{Referências}

1. MOTTA, ANTÔNIO A. et al. Dermatite de contato. Revista Brasileira de Alergia e Imunopatologia, [S. I.], 2011. Disponível em: http://www.asbai.org.br/revistas/vol343/ V34N3- arO1.pdf.

2. PRESA, JULIANA GOMES LOYOLA. Dermatite na região de contato com o vaso

sanitário - avaliação clínico laboratorial. 2014. 162f. Tese ( Doutorado na Saúde da Criança e do Adolescente), Universidade Federal do Paraná. Curitiba, PR. Disponível em: <http:// www.prppg. ufpr.br /ppgped/ sites/ default/ files/Documentos/

dissertacoes_teses/Juliana\%20Loyola\%20quali.pdf>.

3. SOCIEDADE BRASILEIRA DE DERMATOLOGIA. Dermatite de contato: O que é?. [S. I.], 2017. Disponível em: https:// www.sbd.org.br/dermatologia/pele/doencas-eproblemas/ dermatite-de-contato/2/.

4. UCHIDA, CLARICE IZUME. Prevalência das dermatoses ocupacionais no setor de

galvanoplastia registradas no INSS em 2010. Curitiba, 2012. Disponível em: <

http://acervodigital. ufpr. br/ bitstream/ handle/ $1884 / 39040 /$ R\%20-\%20E\%20-\%20

CLARICE $\%$ 20I ZUMI \% 2OUCHIDA. pdf? sequence $=1$ \&isAllowed $=y>$.

5. FERREIRA, BÁRBARA et al. Dermatites: diagnóstico e terapêutica. Brazilian Journal of Surgery and Clinical Research, v, 5,n.2,p.22-26, (Dez 2013 - Fev 2014).

6. NEUMAN MG, NANAU RM, ORUÑA-SANCHEZ L, Coto $G$. Hyaluronic acid and wound healing. J Pharm Pharm Sci. 2015; 18(1):53-60.

7. MONTEIRO, E. O. Tratamento de rejuvenescimento facial com ácido hialurônico não estabilizado de origem não animal aplicado na derme. Moreira Jr. Editora; 198 a 200. Revista Brasileira de Medicina, v.68, n. 6, 2011.

8. XU X, JHA AK, HARRINGTON DA, FARACH-CARSON
MC, JIA X. Hyaluronic Acid-Based Hydrogels: from a Natural Polysaccharide to Complex Networks. Soft Matter. 2012;8(12):3280-3294.

9. SILVA MN, DAMIANI GV, MASSON VA, CALIL SR, VOLPATO V, GONÇALVES $N$, et al. Efeitos do tratamento tópico com ácido hialurônico 0,2\% em queimadura de segundo grau: um relato de experiência. Rev Bras Queimaduras. 2017; 16(1):49-52.

10. SILVA MN, DAMIANI GV, MASSON VA, VOLPATO V, CALIL SR, OLIVEIRA PG, et al. Efetividade do ácido hialurônico $0,2 \%$ em úlcera vasculogênica venosa. Revista Feridas. 2017;05(22):780-788.

11. MASSON VA, SILVA MN, DAMIANI GV, VOLPATO V, CASTILHO JC, OLIVERIA PG. Combinação de ácido hialurônico $02 \%$ e laser de baixa potência em lesões do tipo skin tears. Revista Feridas. 2017;05(27):250-255. 6-8.

12. ANDRADE FSSD, CLARK RMO, FERREIRA ML. Effects of low-level laser therapy on wound healing. Rev Col Bras Cir. 2014 Mar/Apr;41(2):129-33. Doi: http://dx.doi. org/10.1590/S0100-69912014000200010.

13. MATHUR RK, SAHU K, SARAF S, PATHEJA P, KHAN F, GUPTA PK. Low-level laser therapy as an adjunct to conventional therapy in the treatment of diabetic foot ulcers. J Lasers Med Sci. 2017 Feb;32(2):275-82. Doi: 10.1007/ s10103-016-2109-2.

14. BAVARESCO, TALINE et al. Terapia a laser de baixa potência na cicatrização de feridas. Revista de Enfermagem UFPE on line, [S.I.], v. 13, n. 1, p. $216-226$, jan. 2019. ISSN 19818963. Disponível em: <https://periodicos.ufpe.br/revistas/ revistaenfermagem/article/view/235938/31158>. Acesso em: 18 mar. 2021 . doi:https://doi.org/10.5205/19818963-v13ila235938p216-226-2019.

15. RASBAND, W. ImageJ documentation. www.rsb.info. nih.gov 2012. 